Supporting Information

Carbon-based conductive frameworks and metal catalytic sites derived from crosslinked porous porphyrin-based polyimides for enhanced conversion of lithium polysulfides in Li-S batteries

Yongxian Lin a, Junhao Li ${ }^{\text {a }}$, Zhangshi Xiong a, Kaixiang Shi a, *, Quanbing Liu a, *

a Guangzhou Key Laboratory of Clean Transportation Energy Chemistry, School of Chemical Engineering and Light Industry, Guangdong University of Technology, Guangzhou 510006, China.

* Corresponding author. E-mail address: shikx@hotmail.com (K. Shi), liuqb@gdut.edu.cn (Q. Liu)

\title{
Contents:
}

\section{Supplementary method.}

Figure S1. TGA graph of S/CR-Por-H-PPI@800 composite.

Figure S2. FTIR spectra of CR-Por-PPIs and CR-Por-PPIs@800.

Figure S3. Raman spectra of CR-Por-PPIs.

Figure S4. XPS spectra of CR-Por-H-PPI@800 and CR-Por-H-PPI@800-- $\mathrm{Li}_{2} \mathrm{~S}_{6}$.

Figure S5. SEM images of CR-Por-PPIs.

Figure S6. SEM images of S/CR-Por-PPIs@800 composites.

Figure S7. TEM images of CR-Por-PPIs@800.

Figure S8. Nitrogen sorption isotherms and pore size distributions of CR-Por-PPIs.

Figure S9. (a - d) CV curves before cycling for S/CR-Por-PPIs@800 at scan rates from

0.2 to $0.5 \mathrm{mV} \mathrm{s}^{-1}, \mathrm{CV}$ curves peak current of (e) $\mathrm{I}_{\mathrm{A}}$ (anodic oxidation process, $\mathrm{Li}_{2} \mathrm{~S}_{2} / \mathrm{Li}_{2} \mathrm{~S}$ $\rightarrow \mathrm{S}_{8}+\mathrm{Li}$ ), (f) $\mathrm{I}_{\mathrm{C} 1}$ (cathodic reduction process, $\mathrm{S}_{8} \rightarrow \mathrm{Li}_{2} \mathrm{~S}_{\mathrm{x}}, 3 \leqslant \mathrm{x} \leqslant 8$ ), and (g) $\mathrm{I}_{\mathrm{C} 2}$ (cathodic reduction process, $\mathrm{Li}_{2} \mathrm{~S}_{\mathrm{x}} \rightarrow \mathrm{Li}_{2} \mathrm{~S}_{2} / \mathrm{Li}_{2} \mathrm{~S}$ ) for S/CR-Por-PPIs@800 cathodes with multiple effects.

Figure S10. $(\mathrm{a}-\mathrm{d}) \mathrm{CV}$ curves after 500 long-terms cycles at $1 \mathrm{C}$ for S/CR-Por- 
PPIs@800 at scan rates from 0.2 to $0.5 \mathrm{mV} \mathrm{s}^{-1}, \mathrm{CV}$ curves peak current of (e) $\mathrm{I}_{\mathrm{A}}$ (anodic oxidation process, $\mathrm{Li}_{2} \mathrm{~S}_{2} / \mathrm{Li}_{2} \mathrm{~S} \rightarrow \mathrm{S}_{8}+\mathrm{Li}$ ), (f) $\mathrm{I}_{\mathrm{C} 1}$ (cathodic reduction process, $\mathrm{S}_{8} \rightarrow \mathrm{Li}_{2} \mathrm{~S}_{\mathrm{x}}$, $3 \leqslant \mathrm{x} \leqslant 8$ ), and $(\mathrm{g}) \mathrm{I}_{\mathrm{C} 2}$ (cathodic reduction process, $\mathrm{Li}_{2} \mathrm{~S}_{\mathrm{x}} \rightarrow \mathrm{Li}_{2} \mathrm{~S}_{2} / \mathrm{Li}_{2} \mathrm{~S}$ ) for S/CR-PorPPIs@800 cathodes with multiple effects.

Figure S11. Cycling capability and coulombic efficiency of S/CR-Por-H-PPI cathodes at various rates.

Figure S12. Voltage-Capacity curves of S/CR-Por-PPIs@800 composites cathodes at $0.2 \mathrm{C}$.

Figure S13. Voltage-Capacity curves of S/CR-Por-PPIs@800 composites cathodes at $0.5 \mathrm{C}$.

Figure S14. Cycling performance of S/CR-Por-Cu-PPI@800 cathode at 1 C for 1000 cycles.

Figure S15. Voltage-capacity curves of S/CR-Por-PPIs@800 composites cathodes at various rates.

Figure S16. Cycling capability and coulombic efficiency of S/CR-Por-PPIs@800 cathodes at $2 \mathrm{C}$ for long-term cycles.

Table S1 BET surface areas, pore volumes, and pore size distributions within CR-PorPPIs skeletons.

Table S2 Slopes of $\mathrm{I}_{\mathrm{A}}$ (anodic oxidation process, $\mathrm{Li}_{2} \mathrm{~S}_{2} / \mathrm{Li}_{2} \mathrm{~S} \rightarrow \mathrm{S}_{8}+\mathrm{Li}$ ), $\mathrm{I}_{\mathrm{C} 1}$ (cathodic reduction process, $\mathrm{S}_{8} \rightarrow \mathrm{Li}_{2} \mathrm{~S}_{\mathrm{x}}, 3 \leqslant \mathrm{x} \leqslant 8$ ), and $\mathrm{I}_{\mathrm{C} 2}$ (cathodic reduction process, $\mathrm{Li}_{2} \mathrm{~S}_{\mathrm{x}} \rightarrow$ $\mathrm{Li}_{2} \mathrm{~S}_{2} / \mathrm{Li}_{2} \mathrm{~S}$ ) for S/CR-Por-PPIs@800 cathodes with multiple effects.

Table S3 Polarization voltage values of S/CR-Por-PPIs@800 composites hosts based on cyclic voltammetry measurements before cycling at $0.2 \mathrm{mV} \mathrm{s}^{-1}$.

Table S4 Capacity value, $\mathrm{Q}_{\mathrm{SL}}$ and $\mathrm{Q}_{\mathrm{LS}}$ from 0.1 to $1 \mathrm{C}$ based on rate capability.

Table S5 Impedance parameters from S/CR-Por-PPIs@800 composites.

Table S6 Comparison of electrochemical properties of S/CR-Por-PPIs@800 with previously reported metal-based cathode hosts. 


\section{Experimental}

\section{Materials}

Diphenyl sulfone, 1-methyl-2-pyrrolidinone (NMP) and isoquinoline were purchased from Aladdin Industrial. Inc. Copper chloride dehydrate, cobalt chloride hexahydrate, zinc acetate dehydrate, ethanol were provided by Beijing chemical works. All chemical reagents were used as received unless otherwise stated.

\section{Characterization and Methods.}

Fourier transform infrared (FT-IR) spectra were measured on a Nicolet Impact 410 Fourier-transform infrared spectrometer. Powder X-ray diffractions (XRD) using $\mathrm{Cu}$ K $\alpha$ radiation from $4^{\circ}$ to $40^{\circ}$ were carried out on the PANalytical B.V. Empyrean. Raman spectra were performed on a LabRAM HR800 (Horiba). XPS spectra were conducted on Thermo Scientific ${ }^{\mathrm{TM}} \mathrm{K}-\mathrm{Alpha}{ }^{\mathrm{TM}+}$ spectrometer equipped with a monochromatic $\mathrm{Al}$ $\mathrm{K} \alpha$ to analyze the surface chemistry. Field-emission scanning electron microscopy (FESEM) recorded on a SU8020 model HITACHI microscope, and samples were coated with platinum at 20 milliamps prior to use. Transmission electron microscopy (TEM) was collected on a JEOL model JEM-2100 micro-scope. High-resolution transmission electron microscopy (HR-TEM) and energy dispersive X-ray spectroscopy (EDS) were tested by Titan G260-300. Nitrogen sorption isotherms were recorded on a $3 \mathrm{H}-$ 2000PM2 analyzer at $77 \mathrm{~K}$ using Brunauer-Emmett-Teller (BET) model to calculate specific surface areas in the relative pressure $\left(\mathrm{P} / \mathrm{P}_{0}\right)$ of $0.1-0.2$ and original density functional theory (DFT) to collect pore size distribution.

Electrochemical measurements. The S/CR-Por-H-PPI or S/CR-Por-PPIs@800 composites, super $\mathrm{p}$ and polyvinylidene fluoride (PVDF) as weight ratio of $8: 1: 1$ were mingled in N-methyl-2-pyrrolidone (NMP) solution, and then magnetically stirring the mixtures to obtain homogenous slurry. The slurry was cast onto an aluminum foil and dried at $60{ }^{\circ} \mathrm{C}$ under vacuum for $12 \mathrm{~h}$, punching the foil into13.0 mm small disks. Assembling the button battery and using Li foil as the counter electrode, Celgard 2400 
as the separator, $15 \mu \mathrm{L}$ mixture solution $1.0 \mathrm{~mol} \mathrm{~L}^{-1}$ LiTFSI in DOL and DME $(1: 1$ volume ratio) as well as $1.0 \mathrm{wt} \% \mathrm{LiNO}_{3}$ additive as electrolyte. Charge and discharge cycling tests of the lithium-sulfur batteries were recorded at different rate in the voltage range of 1.7 to $2.8 \mathrm{~V}$ at $25{ }^{\circ} \mathrm{C}$ using battery test equipment. We tested the electrochemical impedance spectroscopy (EIS) in the frequency range of $10^{-1}$ to $10^{5}$ Hz. Cyclic voltammograms (CV) tests were conducted on an Auto-Lab electrochemical workstation in the potential range of $1.5-3.0 \mathrm{~V}$ at variable sweeping rate from 0.2 to $0.5 \mathrm{mV} \mathrm{s}^{-1}$.

Symmetrical cell assembly and measurements. The as-fabricated material (CR-PorPPIs@800), super P and PVDF binder according to the weight ratio of 8:1:1 were dispersed in NMP without elemental sulfur. Mixture slurry was homogeneously stirred and then coated on aluminum foil. The $13.0 \mathrm{~mm}$ electrode disks were punched out and used as identical working and counter electrodes. $40 \mu \mathrm{L}$ electrolyte contained $0.5 \mathrm{~mol}$ $\mathrm{L}^{-1} \mathrm{Li}_{2} \mathrm{~S}_{6}$ and $1 \mathrm{~mol} \mathrm{~L}^{-1}$ LiTFSI dissolving in the mixture solution of DOL/DME $(\mathrm{v}: \mathrm{v}=$ 1:1), in which $\mathrm{Li}_{2} \mathrm{~S}_{6}$ was prepared using $\mathrm{Li}_{2} \mathrm{~S}$ and $\mathrm{S}$ as a molar ratio of 1:5. CV measurements of the symmetrical cells were conducted at a scan rate of $50 \mathrm{mV} \mathrm{s}^{-1}$.

Nucleation of Lithium Sulfide. Nucleation of lithium sulfide were tested on commercial carbon paper (CP) current collectors using $\mathrm{Li}_{2} \mathrm{~S}_{8}$ as electrolyte, lithium foil as a counter electrode, Celgard 2400 membrane as separator, in which $\mathrm{Li}_{2} \mathrm{~S}_{8}(25 \mu \mathrm{L}$, $0.25 \mathrm{~mol} / \mathrm{L})$ as catholyte was dropped onto CP-samples, $25 \mu \mathrm{L}$ of LiTFSI (1.0 mol/L) without $\mathrm{Li}_{2} \mathrm{~S}_{8}$ was dropped onto the lithium anode compartment. The cells discharged at $0.112 \mathrm{~mA}$ until the voltage decreased to $2.06 \mathrm{~V}$, and then keeping the voltage at 2.05 $\mathrm{V}$ until the current decreased to $10^{-5} \mathrm{~A}$. During the process of the nucleation and growth of lithium sulphide, their rate of $\mathrm{Li}_{2} \mathrm{~S}$ on different samples were collected and assessed through the energy according to Faraday's law.

\section{Synthesis of porphyrin-based polyimides (Por-H-PPI)}

In a $100 \mathrm{~mL}$ three-necked flask with nitrogen inlet, mechanical stirrer, drying tube and 
constant pressure drop funnel under nitrogen atmosphere, PEPHQDA (1.8099 g, 2.6676 mmol) dissolved in $10 \mathrm{~mL}$ NMP was added, TAPP (0.6 g, $0.8892 \mathrm{mmol})$ dissolving in $10 \mathrm{~mL}$ NMP was added dropwise into the flask. The mixture solution was kept overnight at atmospheric temperature. Subsequently, $2.5 \mathrm{~mL}$ isoquinoline was wadded to facilitate the reaction. The reaction was heated to 120 and $180{ }^{\circ} \mathrm{C}$ and kept for 4 and $24 \mathrm{~h}$, respectively. After cooling to room temperature, the mixture was poured into 200 $\mathrm{mL}$ ethanol to give the precipitate. The precipitate was isolated by filtration and purified in a Soxhlet apparatus for $24 \mathrm{~h}$ with ethanol and dried at $100^{\circ} \mathrm{C}$ under vacuum to afford Por-H-PPI. Yield: $80 \%$.

\section{Synthesis of metalloporphyrin-based polyimides (Por-Cu-PPI, Por-Co-PPI and}

\section{Por-Zn-PPI)}

In a $250 \mathrm{~mL}$ three-necked flask with mechanical stirrer, drying tube and nitrogen inlet under nitrogen atmosphere, Por-H-PPI (0.25 g), $\mathrm{CuCl}_{2}(0.42 \mathrm{~g}, 1.12 \mathrm{mmol})$, and $80 \mathrm{~mL}$ DMF were added. The solution was heated to $100^{\circ} \mathrm{C}$ and kept for $8 \mathrm{~h}$. After cooling to room temperature, the reaction solution was poured into $800 \mathrm{~mL}$ deionized water to give the precipitate. The precipitate was isolated by filtration and washed using deionized water several times. Yield: 92\%.

Por-Co-PPI and Por-Zn-PPI were synthesized according to the same synthetic route of Por-Cu-PPI.

\section{Synthesis of CR-Por-PPIs}

Por-H-PPI was placed in the tube furnace and heated to $360{ }^{\circ} \mathrm{C}$ for $12 \mathrm{~h}$ to afford CRPor-H-PPI. Yield: 64\%.

Other CR-Por-PPIs (CR-Por-Cu-PPI, CR-Por-Co-PPI, CR-Por-Zn-PPI) were prepared using the same synthetic route of CR-Por-H-PPI.

\section{Synthesis of CR-Por-PPIs@800}

CR-Por-H-PPI was placed in the tube furnace and heated to $600{ }^{\circ} \mathrm{C}$ for $2 \mathrm{~h}$, then keeping to $800^{\circ} \mathrm{C}$ for $4 \mathrm{~h}$. Yield: $42 \%$. 
Other CR-Por-PPIs@8000 (CR-Por-Cu-PPI@800,CR-Por-Co-PPI@800,CR-Por-ZnPPI@800) were prepared using the same synthetic route of CR-Por-H-PPI@800.

Synthesis of the Sulfur Composite. The cathode composite was prepared by a meltdiffusion method. Grinding the mixed powder, CR-Por-H-PPI@800 and commercial sulfur (3:7, weight ratio), were added into a $100 \mathrm{~mL}$ Teflon-lined autoclave and heated to $155^{\circ} \mathrm{C}$ for $12 \mathrm{~h}$ to obtain cathode materials. The other composites (S/CR-Por-H-PPI, S/CR-Por-Cu-PPI@800, S/CR-Por-Co-PPI@800, S/CR-Por-Zn-PPI@800) were synthesized according to the same route.

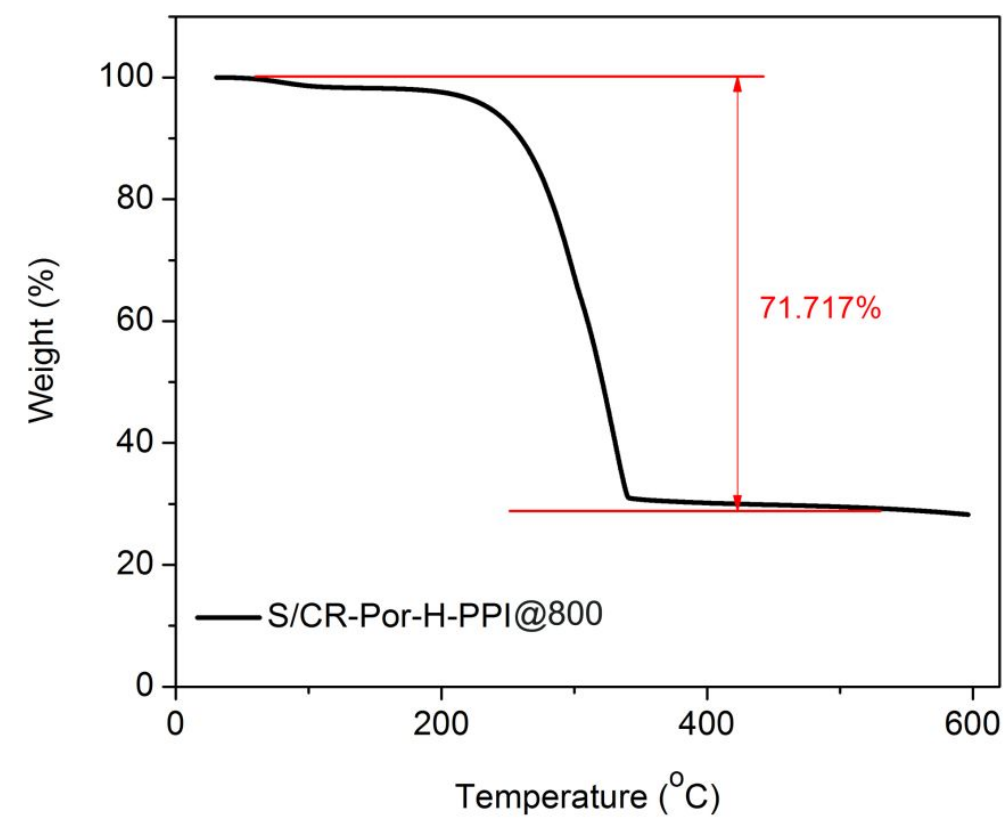

Figure S1. TGA graph of S/CR-Por-H-PPI@800 composite. 

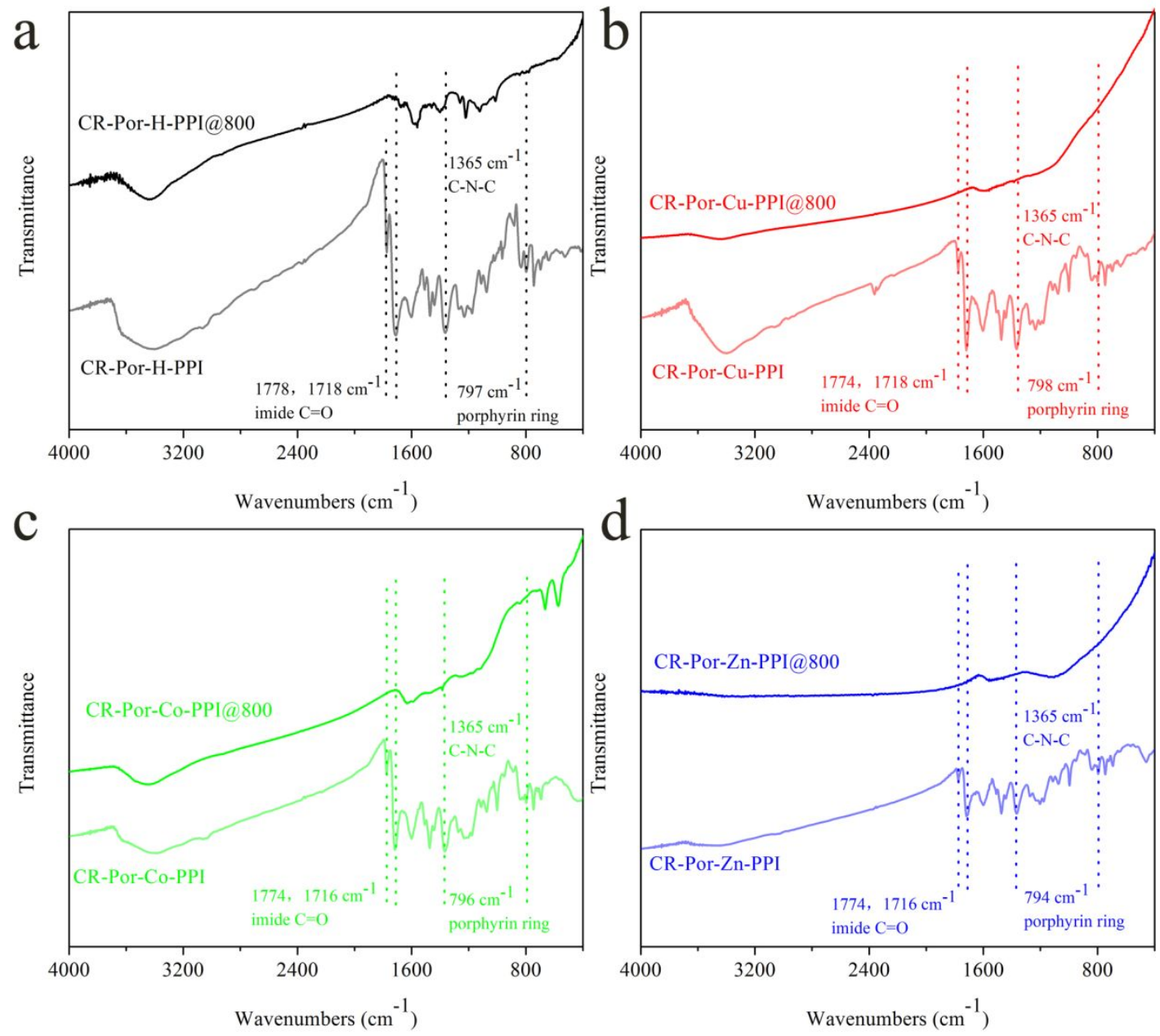

Figure S2. FTIR spectra of CR-Por-PPIs and CR-Por-PPIs@800.

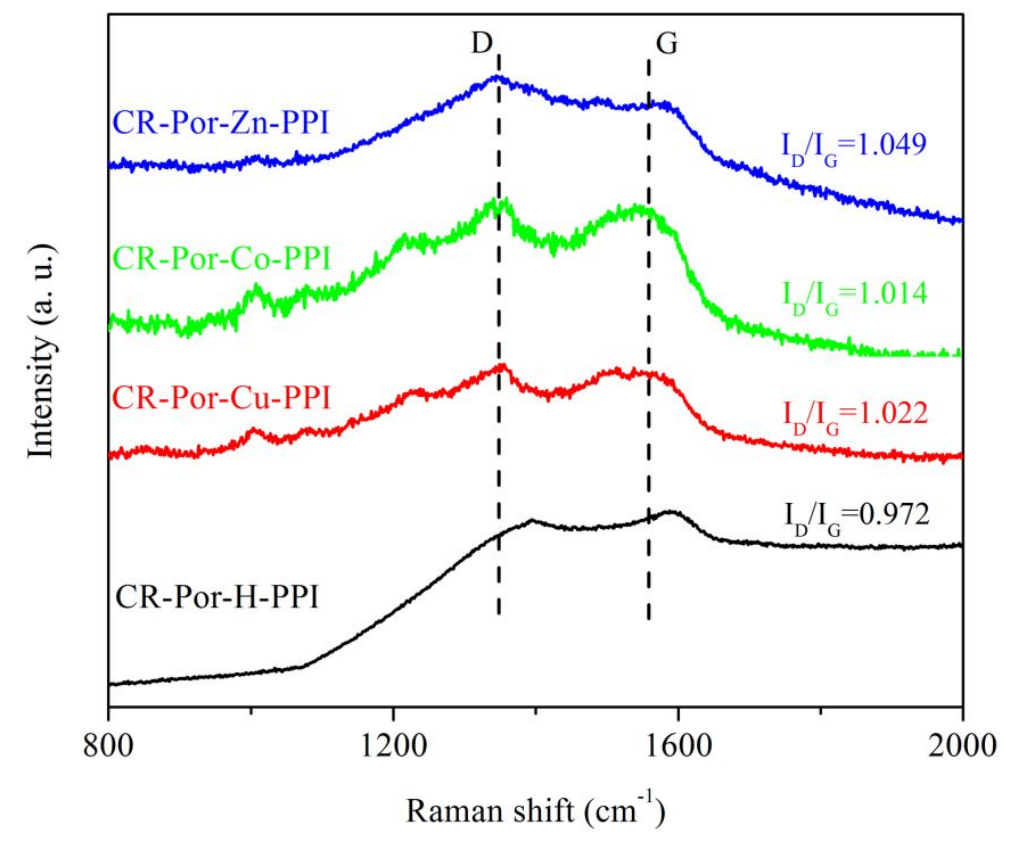

Figure S3. Raman spectra of CR-Por-PPIs. 

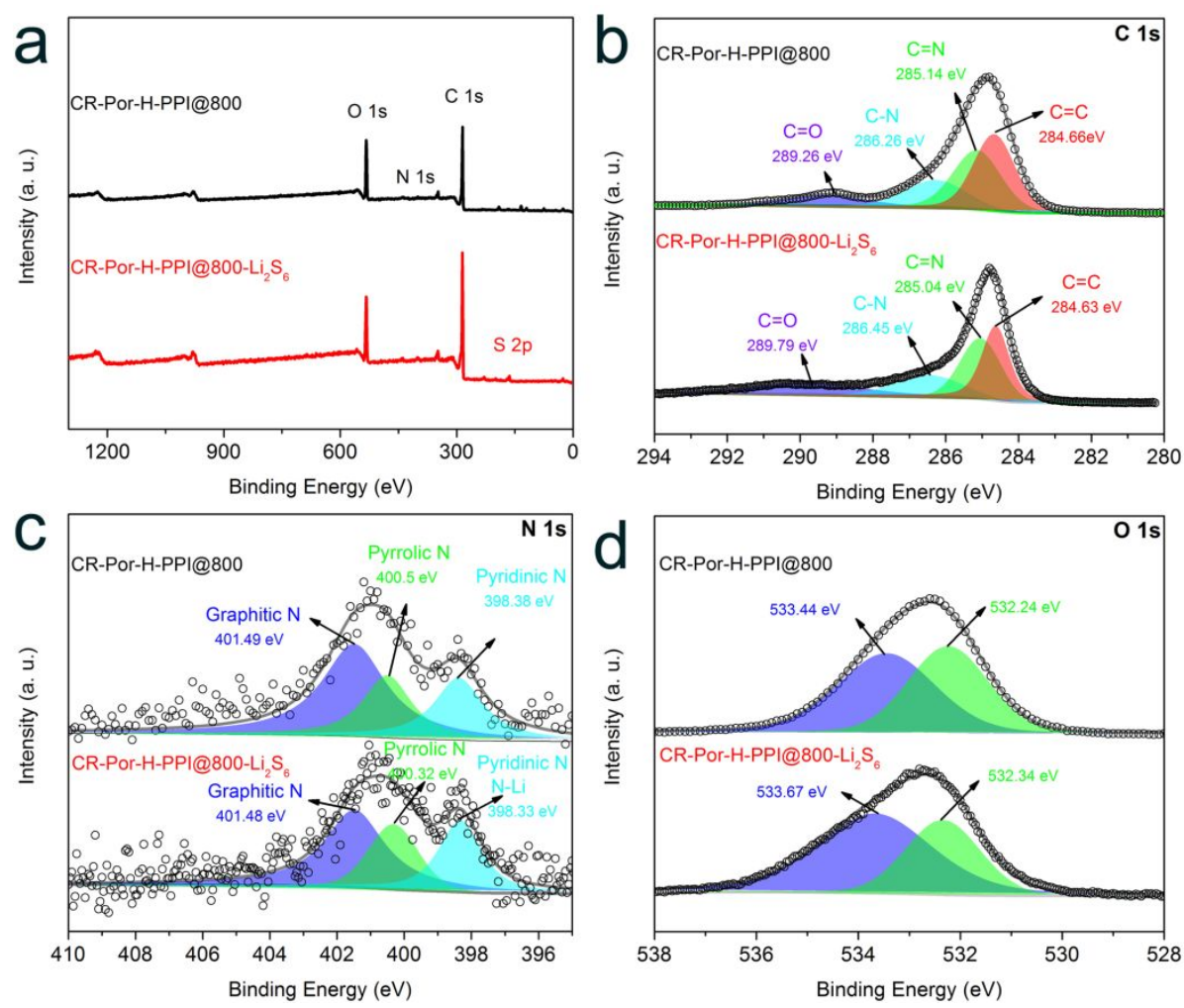

Figure S4. XPS spectra of CR-Por-H-PPI@800 and CR-Por-H-PPI@800-Li $2 \mathrm{~S}_{6}$.

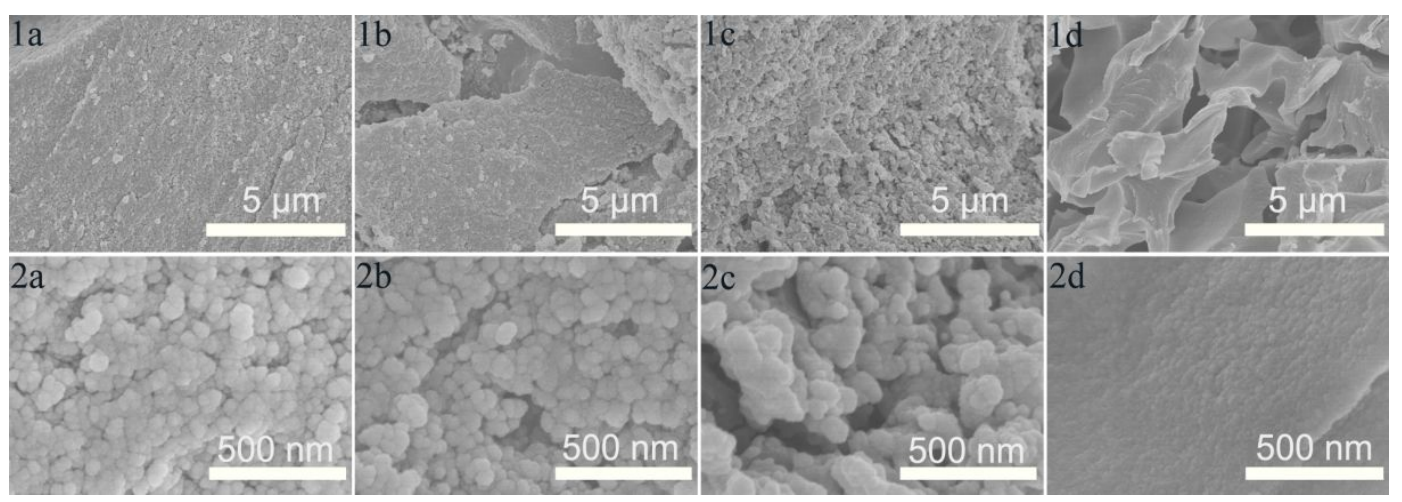

Figure S5. SEM images of CR-Por-H-PPI (1a, 2a), CR-Por-Cu-PPI (1b, 2b), CR-PorCo-PPI (1c, 2c), and CR-Por-Zn-PPI (1d, 2d). 


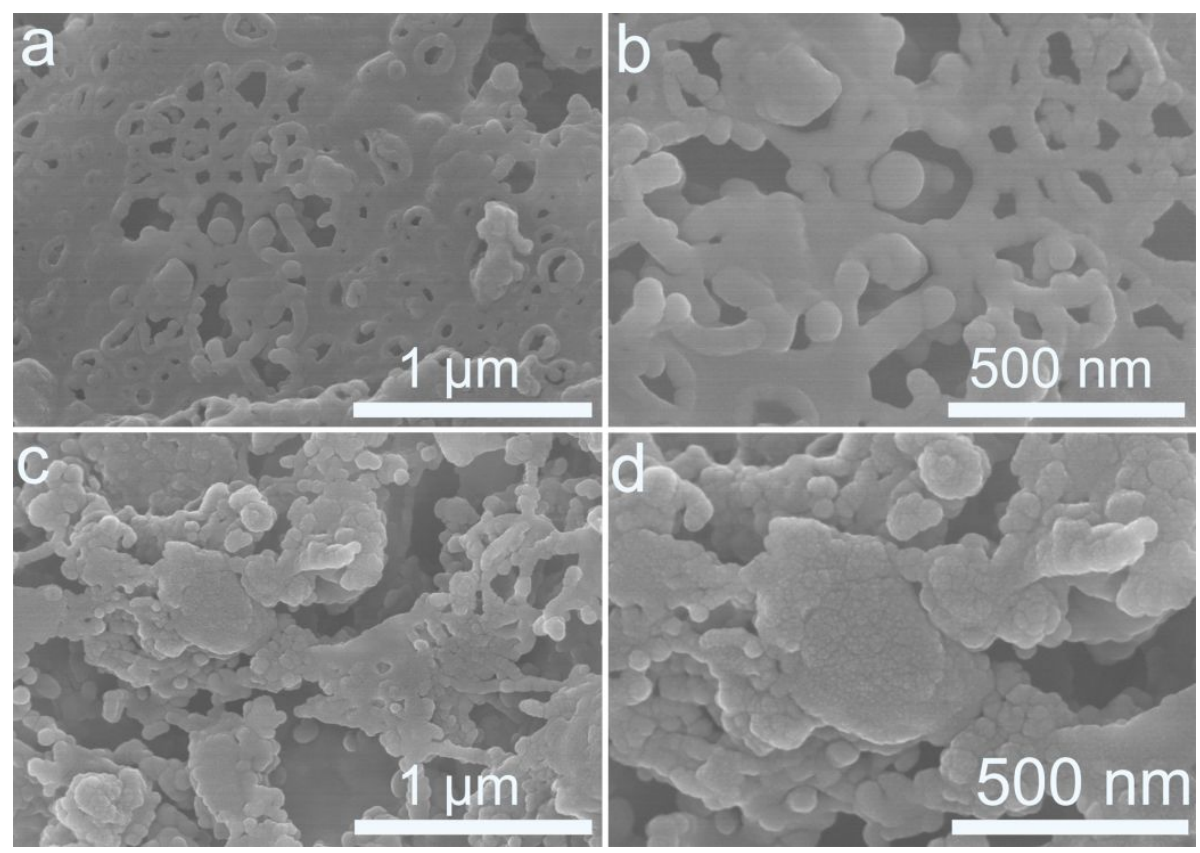

Figure S6. SEM images of S/CR-Por-H-PPI@800 (a, b), S/CR-Por-Cu-PPI@800 (c, d).
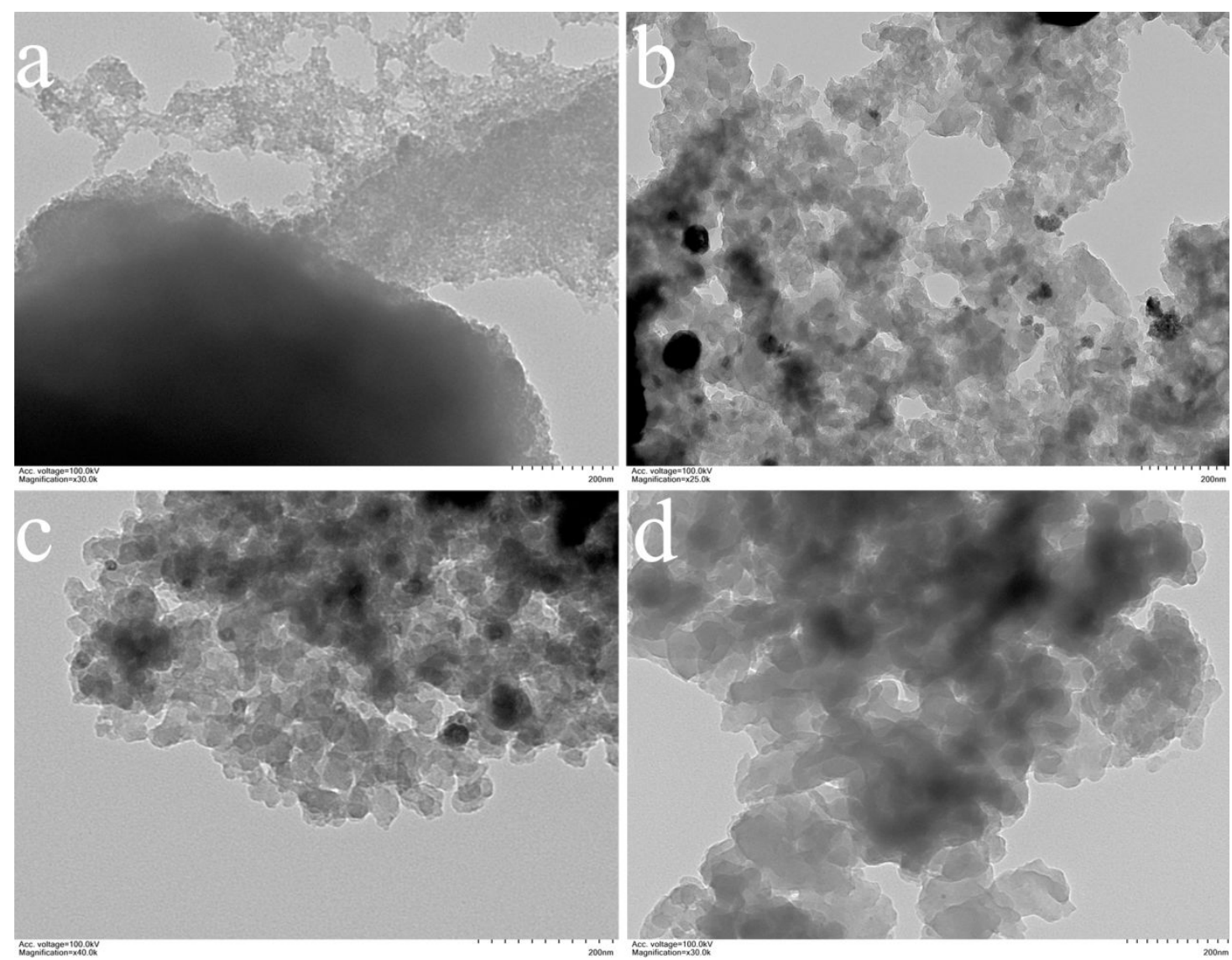

Figure S7. TEM images of (a) CR-Por-H-PPI@800, (b) CR-Por-Cu-PPI@800, (c) CRPor-Co-PPI@800, and (d) CR-Por-Zn-PPI@800. 

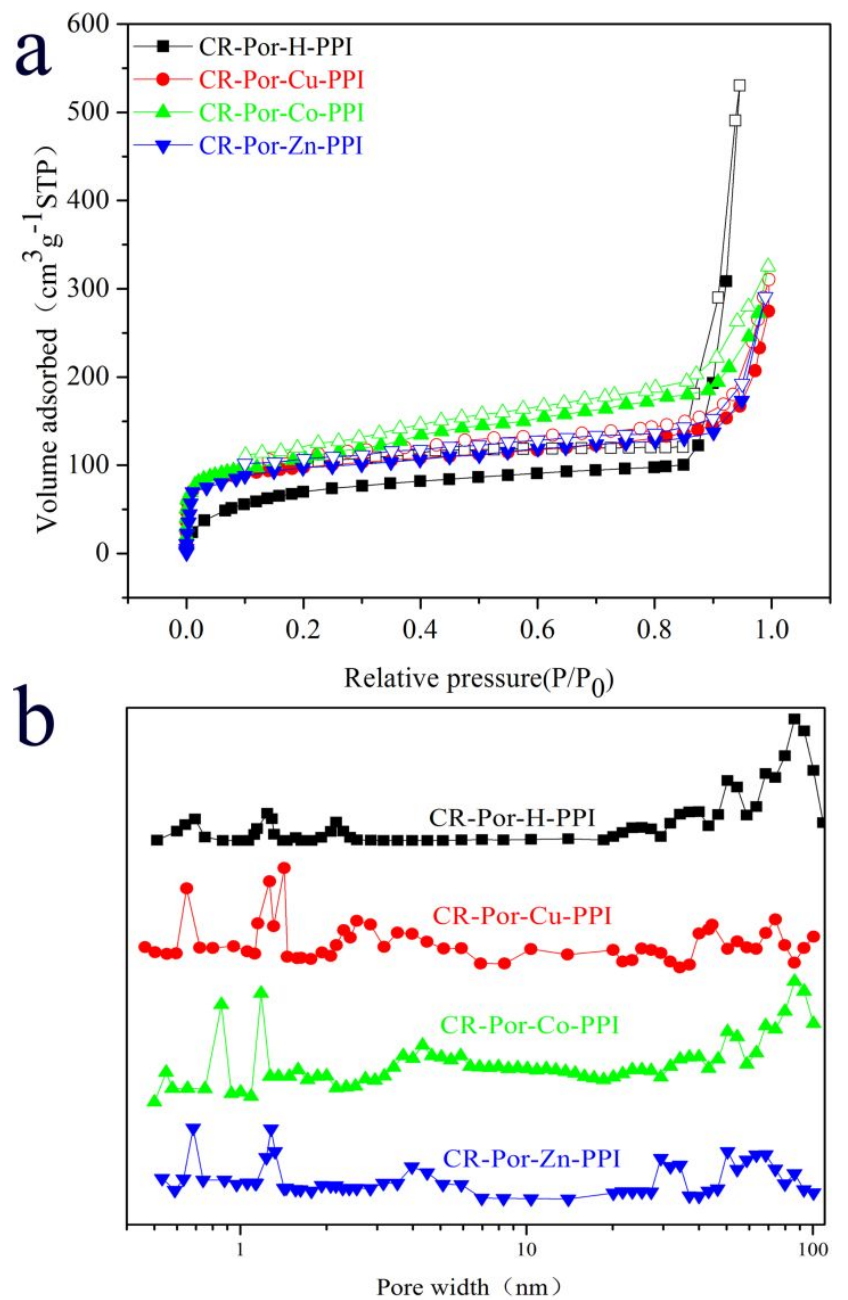

Figure S8. Nitrogen sorption isotherms and pore size distributions of CR-Por-PPIs. 


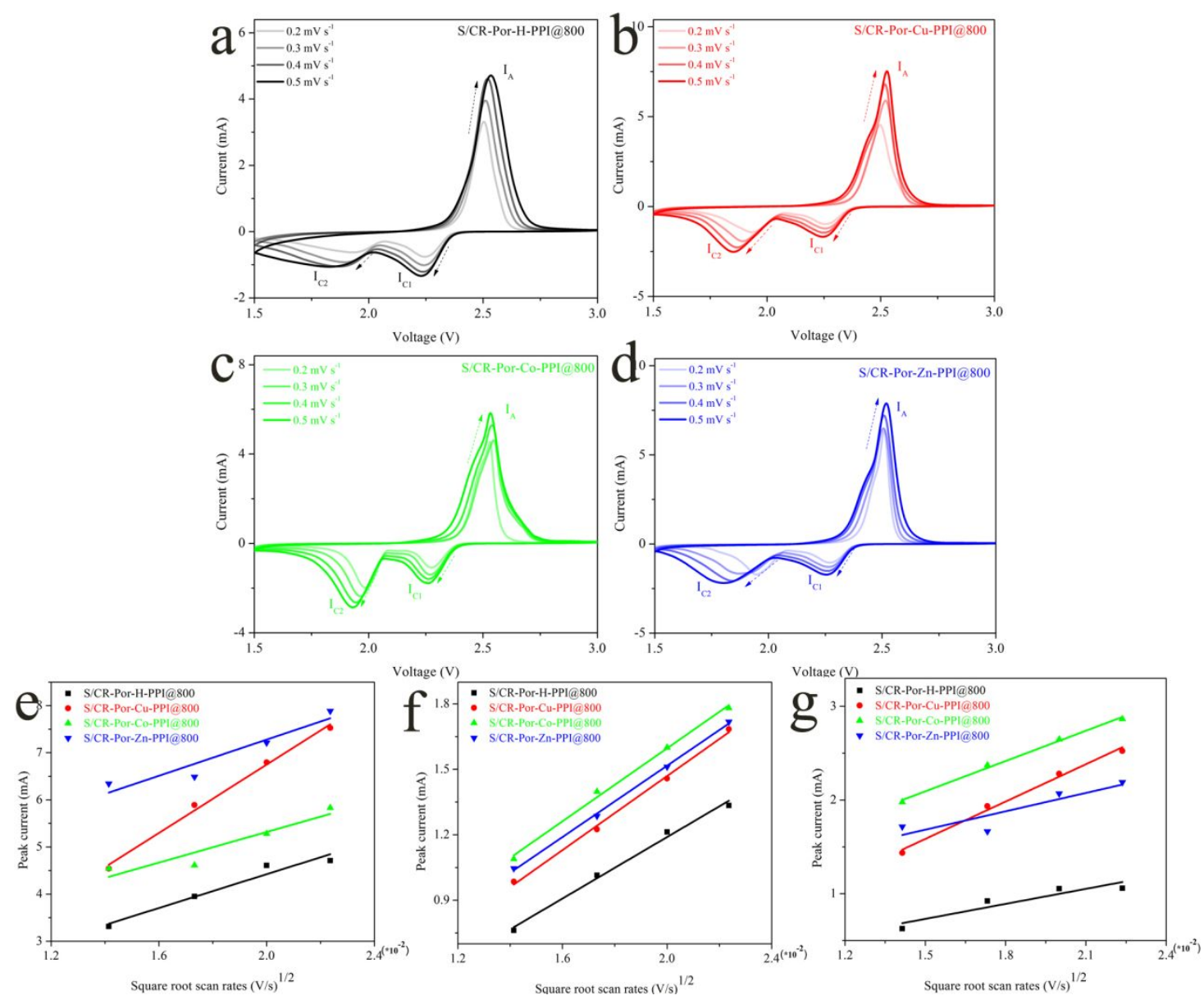

Figure S9. (a - d) CV curves before cycling for S/CR-Por-PPIs@800 at scan rates from 0.2 to $0.5 \mathrm{mV} \mathrm{s}^{-1}, \mathrm{CV}$ curves peak current of (e) $\mathrm{I}_{\mathrm{A}}$ (anodic oxidation process, $\mathrm{Li}_{2} \mathrm{~S}_{2} / \mathrm{Li}_{2} \mathrm{~S}$ $\rightarrow \mathrm{S}_{8}+\mathrm{Li}$ ), (f) $\mathrm{I}_{\mathrm{C} 1}$ (cathodic reduction process, $\mathrm{S}_{8} \rightarrow \mathrm{Li}_{2} \mathrm{~S}_{\mathrm{x}}, 3 \leqslant \mathrm{x} \leqslant 8$ ), and (g) $\mathrm{I}_{\mathrm{C} 2}$ (cathodic reduction process, $\mathrm{Li}_{2} \mathrm{~S}_{\mathrm{x}} \rightarrow \mathrm{Li}_{2} \mathrm{~S}_{2} / \mathrm{Li}_{2} \mathrm{~S}$ ) for S/CR-Por-PPIs@800 cathodes with multiple effects. 

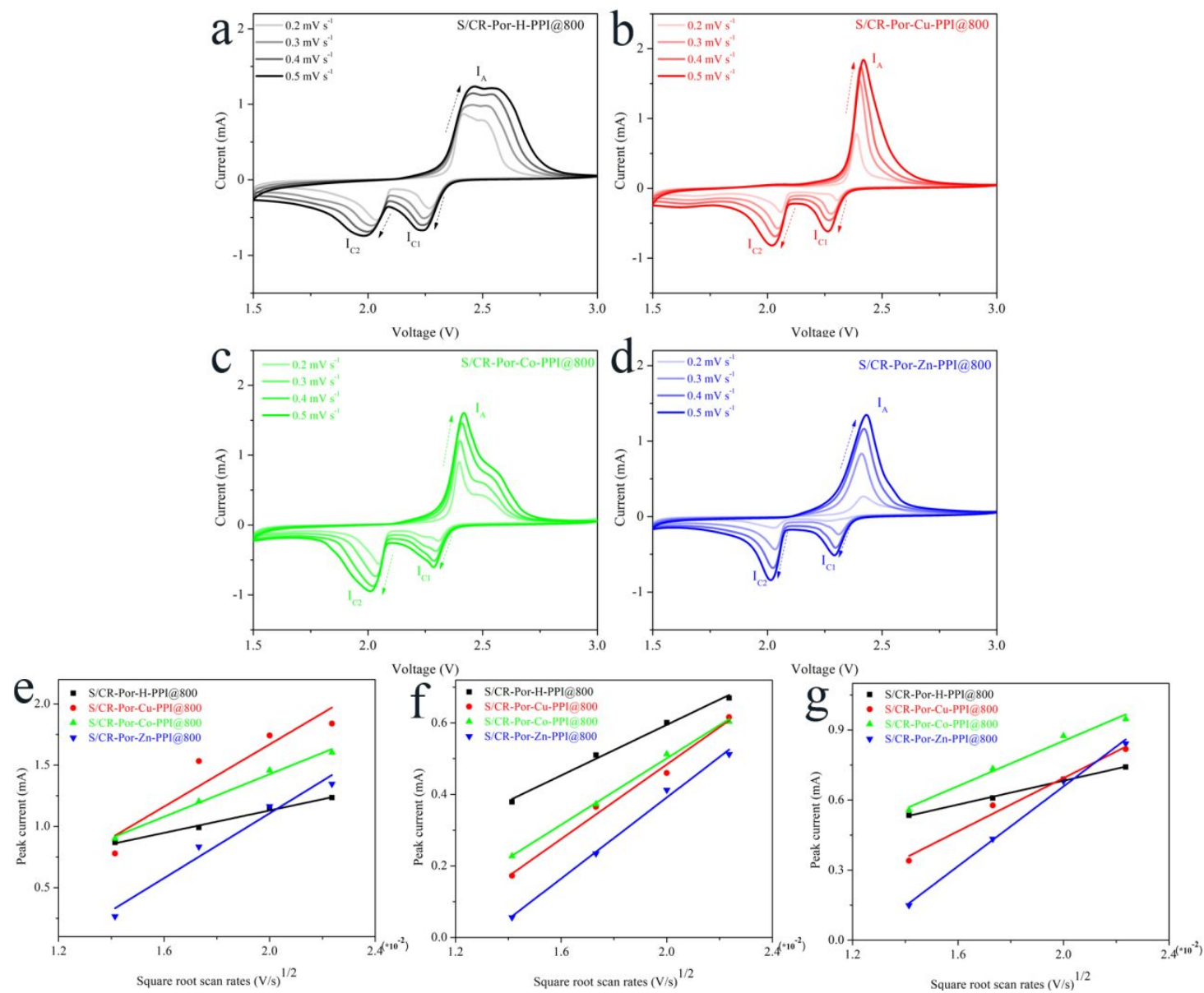

Figure S10. (a - d) CV curves after 500 long-terms cycles at $1 \mathrm{C}$ for S/CR-PorPPIs@800 at scan rates from 0.2 to $0.5 \mathrm{mV} \mathrm{s}^{-1}, \mathrm{CV}$ curves peak current of (e) $\mathrm{I}_{\mathrm{A}}$ (anodic oxidation process, $\mathrm{Li}_{2} \mathrm{~S}_{2} / \mathrm{Li}_{2} \mathrm{~S} \rightarrow \mathrm{S}_{8}+\mathrm{Li}$ ), (f) $\mathrm{I}_{\mathrm{C} 1}$ (cathodic reduction process, $\mathrm{S}_{8} \rightarrow \mathrm{Li}_{2} \mathrm{~S}_{\mathrm{x}}$, $3 \leqslant \mathrm{x} \leqslant 8$ ), and $(\mathrm{g}) \mathrm{I}_{\mathrm{C} 2}$ (cathodic reduction process, $\mathrm{Li}_{2} \mathrm{~S}_{\mathrm{x}} \rightarrow \mathrm{Li}_{2} \mathrm{~S}_{2} / \mathrm{Li}_{2} \mathrm{~S}$ ) for S/CR-PorPPIs@800 cathodes with multiple effects. 

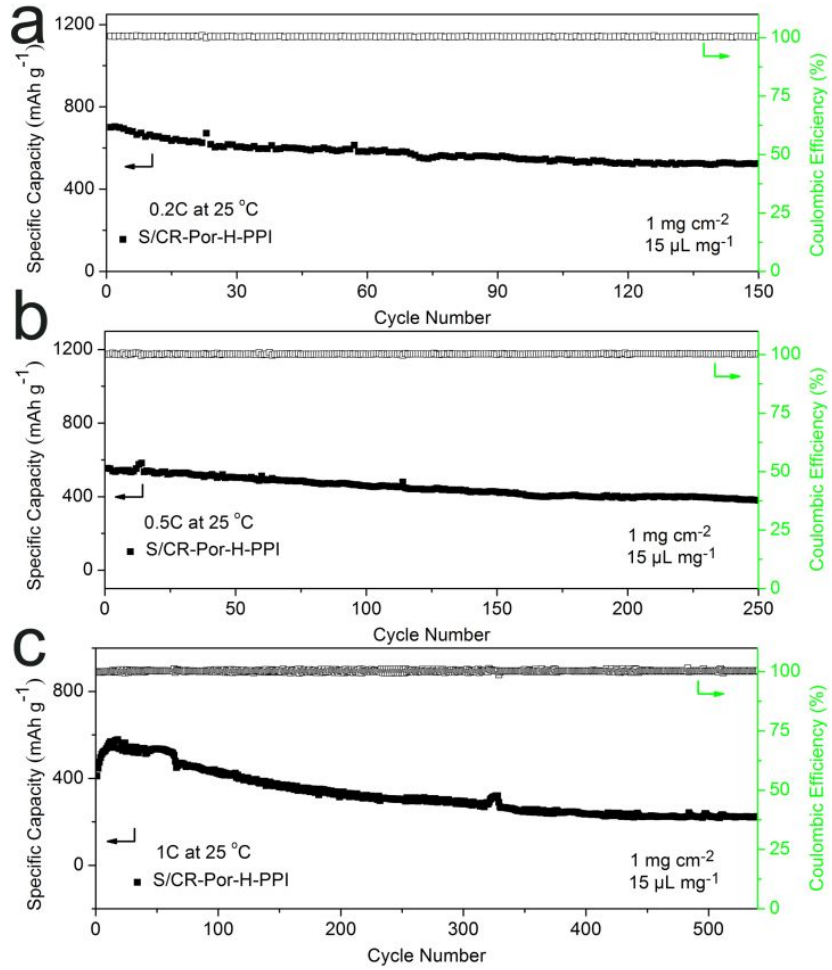

Figure S11. Cycling capability and coulombic efficiency of S/CR-Por-H-PPI cathodes at various rates.
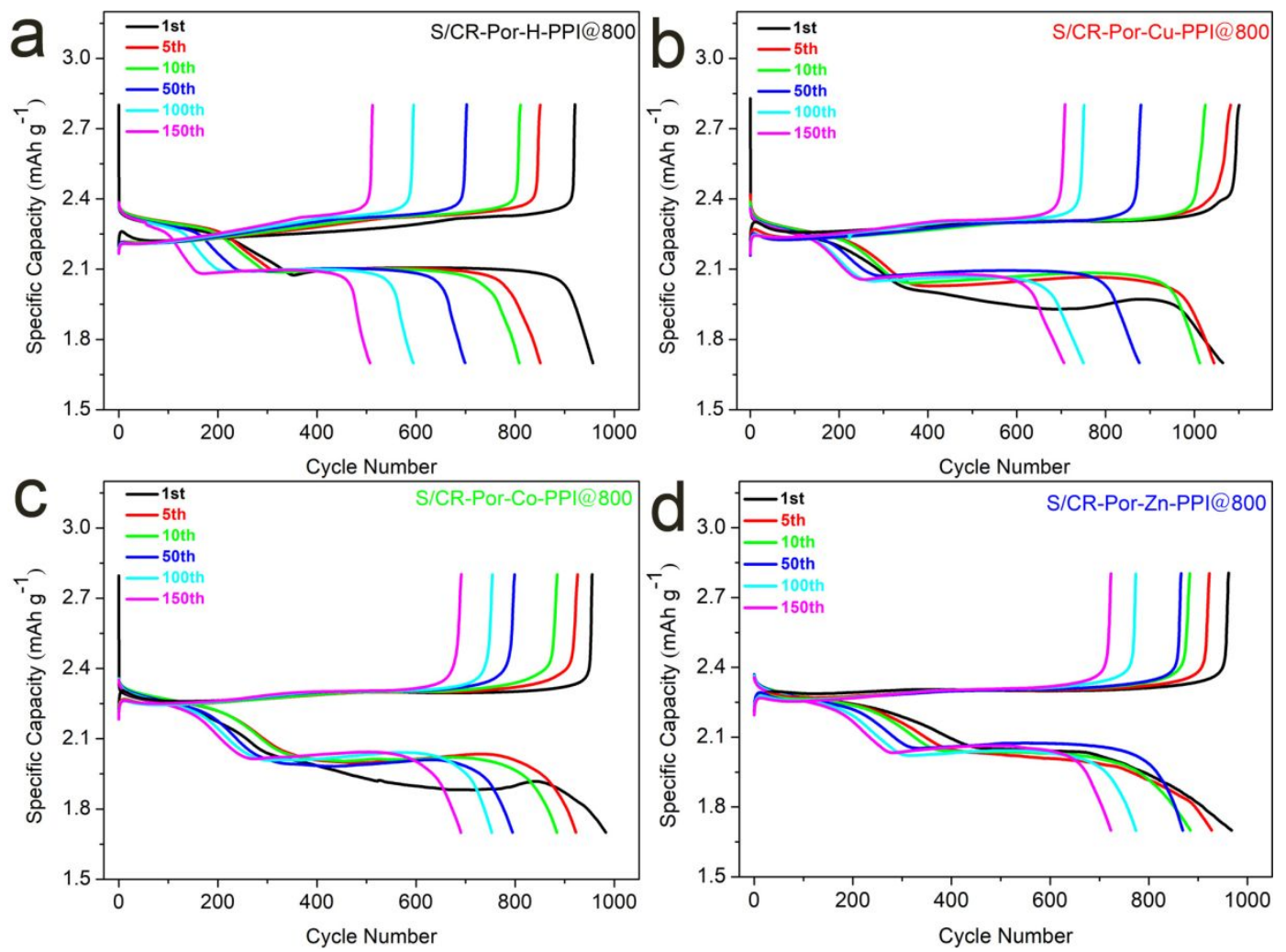

Figure S12. Voltage-Capacity curves of S/CR-Por-PPIs@800 composites cathodes at $0.2 \mathrm{C}$. 

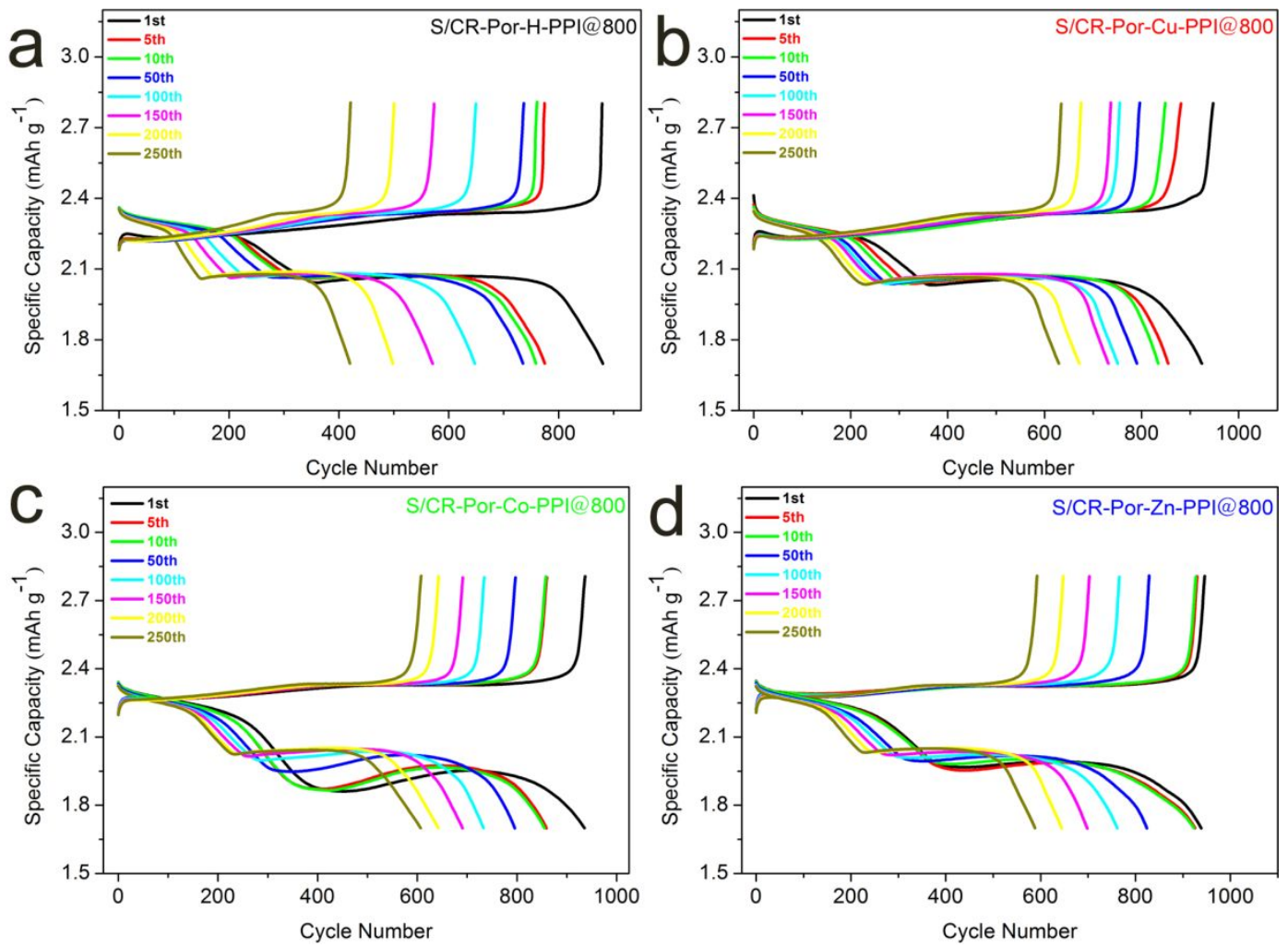

Figure S13. Voltage-Capacity curves of S/CR-Por-PPIs@800 composites cathodes at $0.5 \mathrm{C}$.

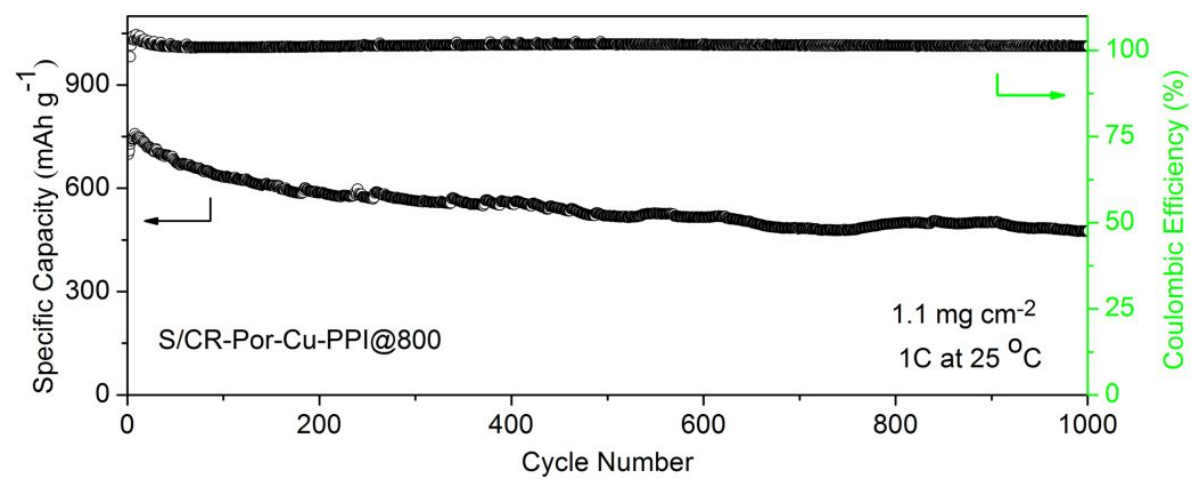

Figure S14. Cycling performance of S/CR-Por-Cu-PPI@800 cathodes at 1 C for 1000 cycles. 

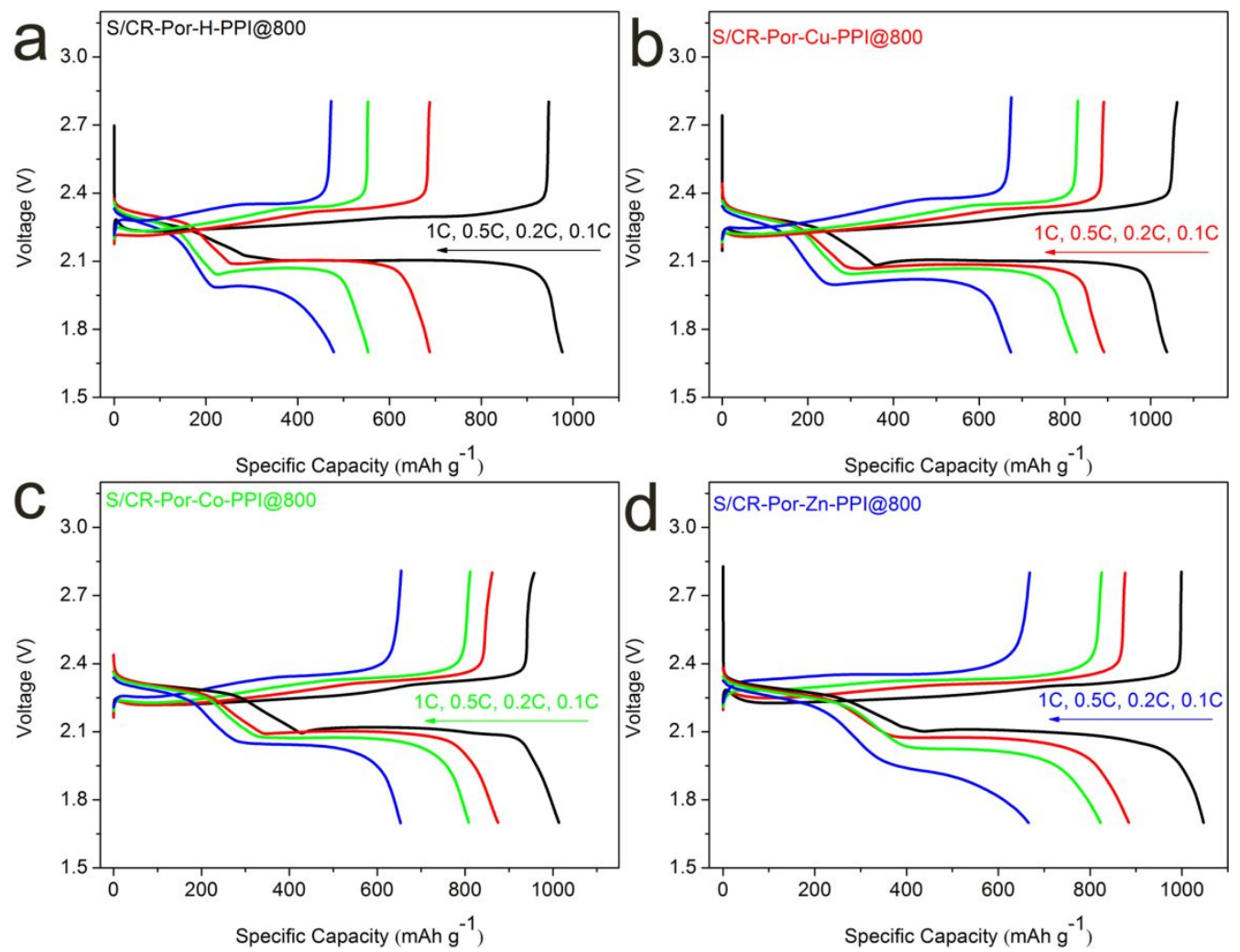

Figure S15. Voltage-capacity curves of S/CR-Por-PPIs@800 composites cathodes at various rates.

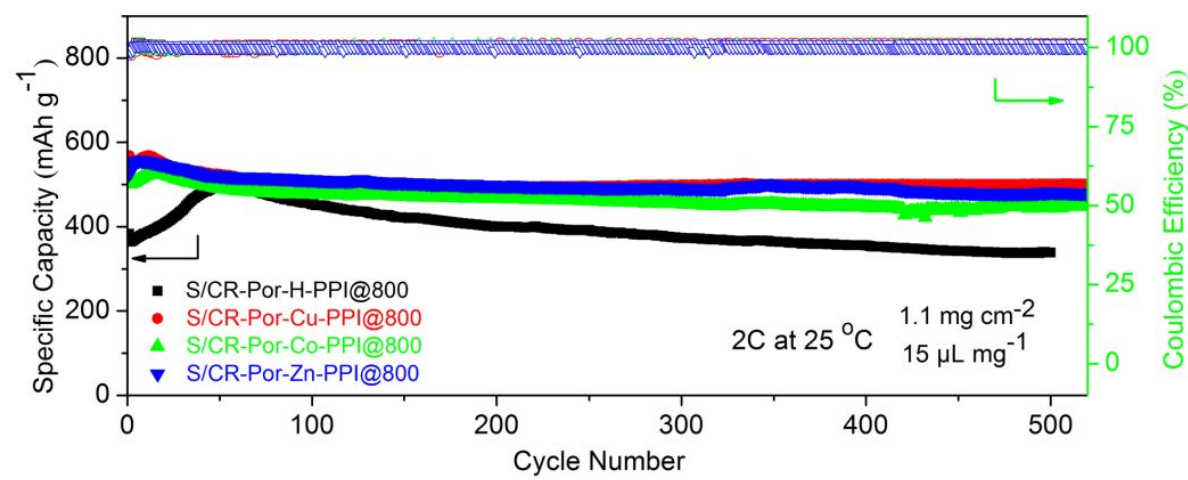

Figure S16. Cycling capability and coulombic efficiency of S/CR-Por-PPIs@800 cathodes at $2 \mathrm{C}$ for long-term cycles. 
Table S1 BET surface areas, pore volumes, and pore size distributions within CR-PorPPIs skeletons.

\begin{tabular}{|c|c|c|c|c|c|c|}
\hline Samples & $\begin{array}{l}S_{\mathrm{BET}^{\mathrm{a}}} \\
\mathrm{m}^{2} \mathrm{~g}^{-1}\end{array}$ & $\begin{array}{c}S_{\text {Langmuir }} \mathrm{b} \\
\mathrm{m}^{2} \mathrm{~g}^{-1}\end{array}$ & $\begin{array}{l}S_{\text {micro }}{ }^{\mathrm{c}} \\
\mathrm{m}^{2} \mathrm{~g}^{-1}\end{array}$ & $\begin{array}{c}V_{\text {total }^{\mathrm{d}}} \\
\mathrm{cm}^{3} \mathrm{~g}^{-1}\end{array}$ & $\begin{array}{c}V_{\text {micro }} \mathrm{e} \\
\mathrm{cm}^{3} \mathrm{~g}^{-1}\end{array}$ & $\begin{array}{c}\text { Pore size }{ }^{f} \\
n m\end{array}$ \\
\hline CR-Por-H-PPI & 261 & 390 & 85 & 0.962 & 0.025 & $\begin{array}{l}0.6,1.2,3- \\
6,30-100\end{array}$ \\
\hline CR-Por-Cu-PPI & 332 & 454 & 170 & 0.480 & 0.080 & $\begin{array}{c}0.6,1.2,3- \\
50\end{array}$ \\
\hline CR-Por-Co-PPI & 350 & 505 & 183 & 0.632 & 0.079 & $\begin{array}{c}0.8,1.2,12- \\
90\end{array}$ \\
\hline CR-Por-Zn-PPI & 320 & 440 & 173 & 0.466 & 0.077 & $\begin{array}{c}0.6,1.2,3- \\
7,27-40\end{array}$ \\
\hline
\end{tabular}

${ }^{a}$ The BET surface area and ${ }^{b}$ Langmuir surface areas of the materials are collected from nitrogen sorption isotherms.

${ }^{\mathrm{c}}$ The micropore surface areas are obtained on the basis of t-plot method.

${ }^{\mathrm{d}}$ Total pore volume comes from the data at $P / P_{0}=0.99$.

${ }^{\mathrm{e}}$ Micropore volume is collected according to t-plot method.

${ }^{\mathrm{f}}$ Pore size distribution is calculated using nonlocal density functional theory. 
Table S2 Slopes of $\mathrm{I}_{\mathrm{A}}$ (anodic oxidation process, $\mathrm{Li}_{2} \mathrm{~S}_{2} / \mathrm{Li}_{2} \mathrm{~S} \rightarrow \mathrm{S}_{8}+\mathrm{Li}$ ), $\mathrm{I}_{\mathrm{C} 1}$ (cathodic reduction process, $\mathrm{S}_{8} \rightarrow \mathrm{Li}_{2} \mathrm{~S}_{\mathrm{x}}, 3 \leqslant \mathrm{x} \leqslant 8$ ), and $\mathrm{I}_{\mathrm{C} 2}$ (cathodic reduction process, $\mathrm{Li}_{2} \mathrm{~S}_{\mathrm{x}} \rightarrow$ $\mathrm{Li}_{2} \mathrm{~S}_{2} / \mathrm{Li}_{2} \mathrm{~S}$ ) for S/CR-Por-PPIs@800 cathodes with multiple effects.

\begin{tabular}{ccccccccc}
\hline & \multicolumn{3}{c}{ The data (before cycling) } & & \multicolumn{3}{c}{ The data (after cycling) } \\
\cline { 2 - 3 } Samples & $\mathbf{I}_{\mathbf{A}}$ & $\mathbf{I}_{\mathbf{C} 1}$ & $\mathbf{I}_{\mathbf{C} 2}$ & & $\mathbf{I}_{\mathbf{A}}$ & $\mathbf{I}_{\mathbf{C} 1}$ & $\mathbf{I}_{\mathbf{C} 2}$ \\
\hline $\begin{array}{c}\text { S/CR-Por-H- } \\
\text { PPI@800 }\end{array}$ & 1.793 & 0.704 & 0.534 & & 0.457 & 0.353 & 0.255 \\
$\begin{array}{c}\text { S/CR-Por-Cu- } \\
\text { PPI@800 }\end{array}$ & 3.623 & 0.848 & 1.328 & & 1.270 & 0.521 & 0.568 \\
$\begin{array}{c}\text { S/CR-Por-Co- } \\
\text { PPI@800 }\end{array}$ & 1.624 & 0.836 & 1.078 & & 0.868 & 0.464 & 0.482 \\
$\begin{array}{c}\text { S/CR-Por-Zn- } \\
\text { PPI@800 }\end{array}$ & 1.918 & 0.817 & 0.654 & & 1.322 & 0.567 & 0.853 \\
\hline
\end{tabular}

Table S3 Polarization voltage values of S/CR-Por-PPIs@800 composites hosts based on cyclic voltammetry measurements before cycling at $0.2 \mathrm{mV} \mathrm{s}^{-1}$.

\begin{tabular}{ccccc}
\hline \multirow{2}{*}{ Samples } & \multicolumn{4}{c}{ Before cycling } \\
\cline { 2 - 5 } & $\mathbf{E}_{\mathbf{A}}(\mathbf{V})$ & $\mathbf{E}_{\mathbf{C 1}}(\mathbf{V})$ & $\mathbf{E}_{\mathbf{C} 2}(\mathbf{V})$ & $\Delta \mathbf{E}(\mathbf{V})$ \\
\hline $\begin{array}{c}\text { S/CR-Por-H- } \\
\text { PPI@800 }\end{array}$ & 2.500 & 2.239 & 1.916 & $0.261 / 0.584$ \\
$\begin{array}{c}\text { S/CR-Por-Cu- } \\
\text { PPI@800 }\end{array}$ & 2.495 & 2.255 & 1.933 & $0.240 / 0.562$ \\
$\begin{array}{c}\text { S/CR-Por-Co- } \\
\text { PPI@800 }\end{array}$ & 2.528 & 2.276 & 1.986 & $0.252 / 0.542$ \\
$\begin{array}{c}\text { S/CR-Por-Zn- } \\
\text { PPI@800 }\end{array}$ & 2.509 & 2.273 & 1.946 & $0.236 / 0.563$ \\
\hline
\end{tabular}


Table S4 Capacity value, $\mathrm{Q}_{\mathrm{SL}}$ and $\mathrm{Q}_{\mathrm{LS}}$ from 0.1 to $1 \mathrm{C}$ based on rate capability.

\begin{tabular}{|c|c|c|c|c|c|c|c|c|c|c|c|c|}
\hline \multirow{2}{*}{ samples } & \multicolumn{3}{|c|}{$0.1 \mathrm{C}$} & \multicolumn{3}{|c|}{$0.2 \mathrm{C}$} & \multicolumn{3}{|c|}{$0.5 \mathrm{C}$} & \multicolumn{3}{|c|}{$1 \mathrm{C}$} \\
\hline & total & $\mathbf{Q}_{\mathrm{SL}}$ & $\mathbf{Q}_{\mathbf{L S}}$ & total & $\mathbf{Q}_{\mathrm{SL}}$ & $\mathbf{Q}_{\mathbf{L S}}$ & total & $\mathbf{Q}_{\mathrm{SL}}$ & $\mathbf{Q}_{\mathbf{L S}}$ & total & $\mathbf{Q}_{\mathrm{SL}}$ & $\mathbf{Q}_{\text {LS }}$ \\
\hline $\begin{array}{c}\text { S/CR-Por-H- } \\
\text { PPI@800 }\end{array}$ & 976 & 301 & 675 & 687 & 258 & 429 & 552 & 224 & 328 & 477 & 219 & 258 \\
\hline $\begin{array}{c}\text { S/CR-Por-Cu- } \\
\text { PPI@800 }\end{array}$ & 1037 & 360 & 677 & 891 & 300 & 591 & 826 & 285 & 541 & 674 & 241 & 433 \\
\hline $\begin{array}{l}\text { S/CR-Por-Co- } \\
\text { PPI@800 }\end{array}$ & 1013 & 429 & 584 & 875 & 346 & 529 & 808 & 326 & 482 & 653 & 282 & 371 \\
\hline $\begin{array}{c}\text { S/CR-Por-Zn- } \\
\text { PPI@ } @ 800\end{array}$ & 1047 & 418 & 629 & 884 & 359 & 525 & 821 & 398 & 423 & 666 & 342 & 324 \\
\hline
\end{tabular}

Table S5 Impedance parameters from S/CR-Por-PPIs@800 composites.

\begin{tabular}{cccccccc}
\hline Samples & \multicolumn{3}{c}{$\begin{array}{c}\text { The data } \\
\text { (before cycling) }\end{array}$} & \multicolumn{4}{c}{$\begin{array}{c}\text { The data } \\
\text { (after cycling) }\end{array}$} \\
\cline { 2 - 9 } & $\mathrm{R}_{\mathrm{ct}^{\mathrm{a}}}{ }^{\mathrm{n}}$ & $\mathrm{R}_{\mathrm{e}}{ }^{\mathrm{b}}$ & $\mathrm{R}_{\text {total }}{ }^{\mathrm{d}}$ & $\mathrm{R}_{\mathrm{ct}}$ & $\mathrm{R}_{\mathrm{e}}$ & $\mathrm{R}_{\mathrm{s}}{ }^{\mathrm{c}}$ & $\mathrm{R}_{\text {total }}$ \\
\hline S/CR-Por-H-PPI@800 & 34.78 & 2.201 & 36.981 & 17.22 & 5.201 & 6.647 & 29.068 \\
S/CR-Por-Cu-PPI@800 & 24.82 & 2.828 & 27.648 & 5.428 & 7.505 & 6.435 & 19.368 \\
S/CR-Por-Co-PPI@800 & 18.81 & 1.785 & 20.595 & 10.93 & 4.547 & 8.053 & 23.53 \\
S/CR-Por-Zn-PPI@800 & 17.96 & 2.271 & 20.231 & 9.866 & 8.811 & 7.412 & 26.089 \\
\hline
\end{tabular}

${ }^{a} R_{c t}$ represent the charge transfer resistance at the electrode/electrolyte interface in high frequency.

${ }^{b} R_{e}$ (the intercept at the real axis $Z^{\prime}$ at high frequency) is the ohmic serial resistance, which comes from the ionic resistance of the electrolyte, the intrinsic resistance of the active materials, and the contact resistance at the active material/current collector interface.

${ }^{c} R_{S}$ is attributed to the solid-electrolyte interface (SEI) film resistance.

${ }^{d} R_{\text {total }}=R_{c t}+R_{e}+R_{S}$.

Table S6 Comparison of electrochemical properties of S/CR-Por-PPIs@800 with previously reported metal-based cathode hosts.

\begin{tabular}{|c|c|c|c|c|c|c|c|}
\hline Host Materials & $\begin{array}{c}\mathrm{S}_{\mathrm{BET}} \\
\mathrm{m}^{2} \mathrm{~g}^{-1}\end{array}$ & $\begin{array}{c}\text { Sulfur } \\
\text { content } \\
\text { wt } \%\end{array}$ & $\begin{array}{l}\text { Areal sulfur } \\
\text { loading } \\
\mathrm{mg} \mathrm{cm}^{-2}\end{array}$ & $\begin{array}{c}\text { Reversible } \\
\text { capacity mAh g }{ }^{-1} \\
\text { (after cycling) }\end{array}$ & $\begin{array}{c}\text { Decay rate } \\
(\%)\end{array}$ & $\begin{array}{l}\text { Cycle } \\
\text { number }\end{array}$ & Ref. \\
\hline $\mathrm{Al}_{2} \mathrm{O}_{3}$ & - & $<56$ & $\sim 1$ & 646 at $0.5 \mathrm{C}$ & 0.180 & 100 & 1 \\
\hline $\mathrm{ZnO}$ & - & 55 & $1.0-1.2$ & 846 at $0.2 \mathrm{C}$ & 0.152 & 100 & 2 \\
\hline $\mathrm{NiO}$ & 880 & 54 & 1 & 714 at $0.2 \mathrm{C}$ & 0.137 & 100 & 3 \\
\hline $\mathrm{Fe}(0.1) / \mathrm{Co}_{3} \mathrm{O}_{4}$ & 44.5 & 77 & - & 1018 at $0.2 \mathrm{C}$ & 0.170 & 150 & 4 \\
\hline $\mathrm{CF} @ \mathrm{CNFs} / \mathrm{MgO}$ & 160 & $49-78$ & 2.4 & 619 at $1 \mathrm{C}$ & 0.091 & 350 & 5 \\
\hline $\mathrm{Fe}_{2} \mathrm{O}_{3}$ & - & $\sim 65$ & 1.2 & 574 at $0.5 \mathrm{C}$ & 0.756 & 50 & 6 \\
\hline $\mathrm{V}_{2} \mathrm{O}_{5}$ & 19.8 & 60 & 1.2 & 838 at $0.1 \mathrm{C}$ & 0.244 & 200 & 7 \\
\hline $\mathrm{WO}_{3}$ & - & - & 5 & 1353 at $0.8 \mathrm{~A} \mathrm{~g}^{-1}$ & 0.010 & 500 & 8 \\
\hline $\mathrm{MoSe}_{2} / \mathrm{MoO}_{2}$ & 104 & 66 & - & 848 at $0.5 \mathrm{C}$ & 0.045 & 500 & 9 \\
\hline $\mathrm{SnO}_{2} @ \mathrm{TMS}$ & 108 & 70 & 2.75 & 1175 at $0.5 \mathrm{C}$ & 0.034 & 200 & 10 \\
\hline $\mathrm{Nb}_{2} \mathrm{O}_{5} / \mathrm{RGO}$ & 287 & 50 & $0.1-0.5$ & 628 at $3 \mathrm{~A} \mathrm{~g}^{-1}$ & 0.046 & 500 & 11 \\
\hline NiMoO4@NSCC & - & - & 2 & 997 at $0.2 \mathrm{C}$ & 0.179 & 80 & 12 \\
\hline $\mathrm{Fe}_{3} \mathrm{O}_{4}$ & 47.1 & 46.6 & 1.73 & 674 at $0.2 \mathrm{C}$ & 0.140 & 100 & 13 \\
\hline $\begin{array}{l}\text { CR-Por-H- } \\
\text { PPI@800 }\end{array}$ & 504 & 56 & 1.1 & 354 at $1 \mathrm{C}$ & 0.081 & 500 & $\begin{array}{l}\text { This } \\
\text { work }\end{array}$ \\
\hline $\begin{array}{l}\text { CR-Por-Cu- } \\
\text { PPI@800 }\end{array}$ & 551 & 56 & 1.1 & 520 at $1 \mathrm{C}$ & 0.051 & 500 & \\
\hline $\begin{array}{c}\text { CR-Por-Co- } \\
\text { PPI@800 }\end{array}$ & 573 & 56 & 1.1 & 469 at $1 \mathrm{C}$ & 0.066 & 500 & \\
\hline $\begin{array}{c}\text { CR-Por-Zn- } \\
\text { PPI@800 }\end{array}$ & 548 & 56 & 1.1 & 509 at $1 \mathrm{C}$ & 0.070 & 500 & \\
\hline
\end{tabular}




\section{References}

(1) Yu, M.; Yuan, W.; Li, C.; Hong, J.-D.; Shi, G. Performance Enhancement of a Graphene-Sulfur Composite as a Lithium-Sulfur Battery Electrode by Coating with an Ultrathin $\mathrm{Al}_{2} \mathrm{O}_{3}$ Film Via Atomic Layer Deposition. J. Mater. Chem. A 2014, 2, 73607366.

(2) Yu, M.; Wang, A.; Tian, F.; Song, H.; Wang, Y.; Li, C.; Hong, J. D.; Shi, G. DualProtection of a Graphene-Sulfur Composite by a Compact Graphene Skin and an Atomic Layer Deposited Oxide Coating for a Lithium-Sulfur Battery. Nanoscale 2015, 7, 5292-5298.

(3) Chen, C.; Xu, H.; Zhang, B.; Jiang, Q.; Zhang, Y.; Li, L.; Lin, Z. Rational Design of a Mesoporous Silica-Based Cathode for Efficient Trapping of Polysulfides in Li-S Batteries. Chem. Commun. 2020, 56, 786-789.

(4) Wang, W.; Zhao, Y.; Zhang, Y.; Wang, J.; Cui, G.; Li, M.; Bakenov, Z.; Wang, X. Defect-Rich Multishelled Fe-Doped $\mathrm{Co}_{3} \mathrm{O}_{4}$ Hollow Microspheres with Multiple Spatial Confinements to Facilitate Catalytic Conversion of Polysulfides for High-Performance Li-S Batteries. ACS Appl. Mater. Interfaces 2020, 12, 12763-12773.

(5) Xiang, M.; Wu, H.; Liu, H.; Huang, J.; Zheng, Y.; Yang, L.; Jing, P.; Zhang, Y.; Dou, S.; Liu, H. A Flexible 3d Multifunctional Mgo-Decorated Carbon Foam@Cnts Hybrid as Self-Supported Cathode for High-Performance Lithium-Sulfur Batteries. $A d v$. Funct. Mater. 2017, 27, 1702573.

(6) Zhao, C.; Shen, C.; Xin, F.; Sun, Z.; Han, W. Prussian Blue-Derived $\mathrm{Fe}_{2} \mathrm{O}_{3} / \mathrm{Sulfur}$ Composite Cathode for Lithium-Sulfur Batteries. Mater. Lett. 2014, 137, 52-55.

(7) Wang, C.; Yi, Y.; Li, H.; Wu, P.; Li, M.; Jiang, W.; Chen, Z.; Li, H.; Zhu, W.; Dai, S. Rapid Gas-Assisted Exfoliation Promises $\mathrm{V}_{2} \mathrm{O}_{5}$ Nanosheets for High Performance Lithium-Sulfur Batteries. Nano Energy 2020, 67, 104253.

(8) Choi, S.; Seo, D. H.; Kaiser, M. R.; Zhang, C.; van der laan, T.; Han, Z. J.; Bendavid, A.; Guo, X.; Yick, S.; Murdock, A. T.; Su, D.; Lee, B. R.; Du, A.; Dou, S. 
X.; Wang, G. $\mathrm{WO}_{3}$ Nanolayer Coated 3d-Graphene/Sulfur Composites for High Performance Lithium/Sulfur Batteries. J. Mater. Chem. A 2019, 7, 4596-4603.

(9) Hao, Q.; Cui, G.; Zhang, Y.; Li, J.; Zhang, Z. Novel $\mathrm{MoSe}_{2} / \mathrm{MoO}_{2}$ Heterostructure as an Effective Sulfur Host for High-Performance Lithium/Sulfur Batteries. Chem. Eng. J. 2020, 381, 122672.

(10)Wang, M.; Fan, L.; Tian, D.; Wu, X.; Qiu, Y.; Zhao, C.; Guan, B.; Wang, Y.; Zhang, N.; Sun, K. Rational Design of Hierarchical $\mathrm{SnO}_{2} / 1 \mathrm{t}-\mathrm{MoS}_{2}$ Nanoarray Electrode for Ultralong-Life Li-S Batteries. ACS Energy Lett. 2018, 3, 1627-1633.

(11)Guo, P.; Sun, K.; Shang, X.; Liu, D.; Wang, Y.; Liu, Q.; Fu, Y.; He, D. $\mathrm{Nb}_{2} \mathrm{O}_{5} / \mathrm{Rgo}$ Nanocomposite Modified Separators with Robust Polysulfide Traps and Catalytic Centers for Boosting Performance of Lithium-Sulfur Batteries. Small 2019, 15, 1902363.

(12)Sun, T.; Zhao, X.; Li, B.; Shu, H.; Luo, L.; Xia, W.; Chen, M.; Zeng, P.; Yang, X.; Gao, P.; Pei, Y.; Wang, X. NiMoO 4 Nanosheets Anchored on N-S Doped Carbon Clothes with Hierarchical Structure as a Bidirectional Catalyst toward Accelerating Polysulfides Conversion for Li-S Battery. Adv. Funct. Mater. 2021, 31, 2101285.

(13)Zhou, T.; Shen, Z.; Wu, Y.; Han, T.; Zhu, M.; Qiao, X.; Zhu, Y.; Zhang, H.; Liu, J. A Yolk-Shell $\mathrm{Fe}_{3} \mathrm{O}_{4} @$ Void@Carbon Nanochain as Shuttle Effect Suppressive and Volume-Change Accommodating Sulfur Host for Long-Life Lithium-Sulfur Batteries. Nanoscale 2021, 13, 7744-7750. 